\title{
Isolated Fallopian Tube Torsion: A Rare Occurrence
}

\author{
Nidhi Jain ${ }^{1^{*}}$, Rahul Manchanda ${ }^{2}$, Sravani Chithra ${ }^{1}$, Anshika lekhi ${ }^{1}$ \\ ${ }^{1}$ Fellow in Gynae endoscopy, ${ }^{2} \mathrm{HOD}$, Department of Gynae endoscopy, Manchanda's Endoscopic Centre, PSRI, Delhi, INDIA.
}

\begin{abstract}
Isolated fallopian tube torsion [IFTT] is one of the rare gynaecological causes of acute abdomen. Its incidence is reported as 1:15,00,000. However, it is one of the most challenging entities for the clinicians to diagnose due to absence of pathognomic symptoms, signs and investigations. It is most commonly seen in women of reproductive age group. However, no age is exempted from it. With this background, authors here report a case of 13-year-old girl who presented to casualty of our hospital with acute lower abdominal pain on right side since two days. She was not sexually active. Her blood and urine investigations were normal. Ultrasound pelvis revealed a right adnexal cyst of size $5 \times 6 \mathrm{~cm}$. Urgent laparoscopy was done which showed IFTT of right side with bilateral paratubal cyst. Laparoscopic detorsion with bilateral cystectomy was done. On conclusion, early diagnosis and management of IFTT is essential to salvage the tubes. Laparoscopy is considered as gold standard for its diagnosis and simultaneous management. Conservative therapy
\end{abstract}

\section{INTRODUCTION}

Isolated fallopian tube torsion [IFTT] is defined as isolated torsion of fallopian tube in absence of ovarian torsion. It is one of the rare gynaecological causes of acute abdomen. Incidence of isolated fallopian tube torsion is 1 in 1.5 million women ${ }^{1}$. It is most commonly seen in women of reproductive age group. The most common presenting feature is acute onset of pelvic pain however there is no pathognomic symptoms and signs for its diagnosis, making its diagnosis a challenge for the clinician. Prompt intervention is necessary to diagnose and treat the pathology so as to preserve the reproductive function. With this background, authors here report a case of 13 year old girl who presented with acute abdomen and was found to have IFTT on diagnostic laparoscopy.

\section{CASE}

A 13-year-old girl came to casualty of our hospital with acute pain in the lower abdomen on right side since two days. There was no complaint of nausea, vomiting or fever. There was no complaint of burning during micturition or altered bowel habits. She had achieved her menarche 1 year back. She was having regular menstrual cycle of 28 days, lasting for 5 days, with average blood flow and without complaint of dysmenorrhea. Her last period was twenty days prior to admission. She was not sexually active. On physical examination, patient was haemodynamically stable. On palpation, abdominal guarding and tenderness was present in right iliac fossa.

Complete blood count and urine analysis was done, which was normal. Ultrasound examination was done which revealed a right adnexal cyst of size $5 \times 6 \mathrm{~cm}$ without internal septations or including detorsion with cystectomy should be considered as first line treatment. Authors report this case due to its rarity.

Keywords: Abdominal pain, Detorsion, Fallopian tube. *Correspondence to:

Dr. Nidhi Jain, House no. 655, Police line area, Near S.S.P. residence, Hisar, Haryana.

Email: nidhijain270587@gmail.com

Article History:

Received: 02-05-2016, Revised: 05-05-2016, Accepted: 06-05-2016

\begin{tabular}{|l|c|}
\hline \multicolumn{2}{|c|}{ Access this article online } \\
\hline Website: & Quick Response code \\
www.ijmrp.com & \\
\hline DOI: & \\
10.21276/ijmrp.2016.2.3.046 & \\
\hline
\end{tabular}

calcifications. In view of acute onset of pain, physical finding, and detection of an adnexal mass on ultrasonography, provisional diagnosis of right adnexal torsion was made and thus, urgent laparoscopy was performed.

On laparoscopy, right sided isolated fallopian tube torsion (Fig 1) was found with two and half turns along with bilateral paratubal cysts (Fig 2) of size $7 \mathrm{~cm}$ on right side and $3 \mathrm{~cm}$ on left side. Normal ovary was visualized on right side. Detorsion of tube was done followed by bilateral paratubal cystectomy. Specimen was sent for histopathological examination, which was reported as benign paratubal cyst (Fig 3). Following surgery, patient was well and discharged on next day of surgery.

\section{DISCUSSION}

Isolated fallopian tube torsion is defined as the twisting of the fallopian tube by at least one complete turnaround a centre-line consisting of the infundibulopelvic ligament or tubo-ovarian ligament ${ }^{2}$. It was first described by Bland-Sutton T. ${ }^{3}$ in 1890 as a rare cause of lower abdominal pain.

The aetiology of IFTT is not well known. Several risk factors have been identified such as hyper motility of tube, hydrosalpinx, tubal adhesions, prior surgery such as tubal ligation, ovarian or paraovarian mass, pregnancy or malignancy. Tubal torsion is defined as primary when it is not associated with any of these risk factors, being idiopathic in aetiology and secondary if associated with underlying pathology. In the case reported here, torsion was secondary in origin being associated with paratubal cyst.

It primarily affects women of reproductive age group. However, it can be seen even in prepubertal girls and postmenopausal 
women. In 2013, Casey RK et al ${ }^{4}$ did a retrospective study to review fifteen cases of IFTT in pediatric age group, in which youngest child who was diagnosed with IFTT was 8 years old while in another case report by Djing et al ${ }^{5}$ in 2007 , a 70 yr old woman was diagnosed with IFTT. Here, we have reported IFTT in a 13 years old girl who has attained her menarche.

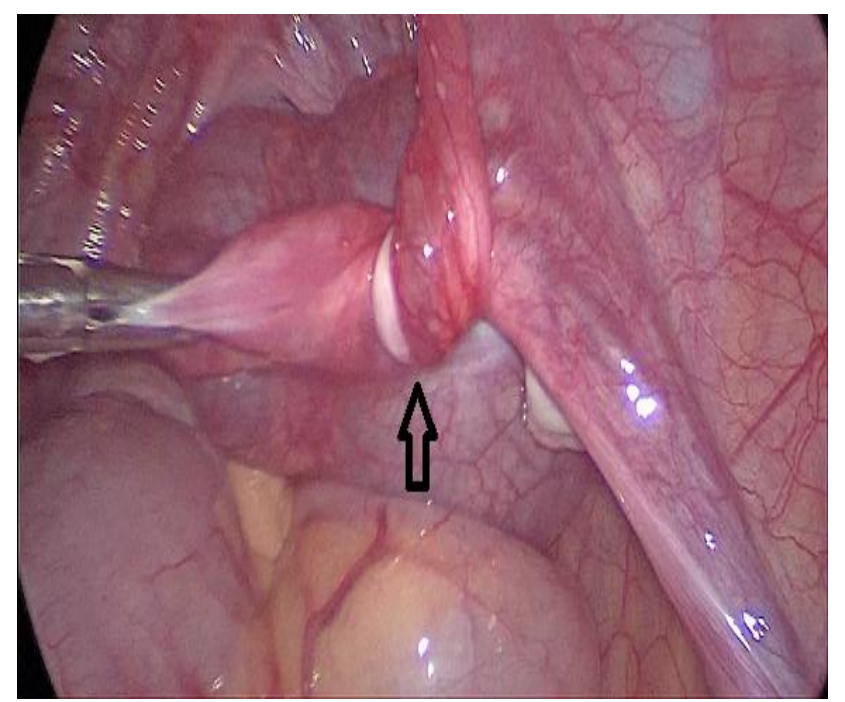

Fig 1: laparoscopic view of pelvis with arrow pointing towards isolated fallopian tube torsion of right side

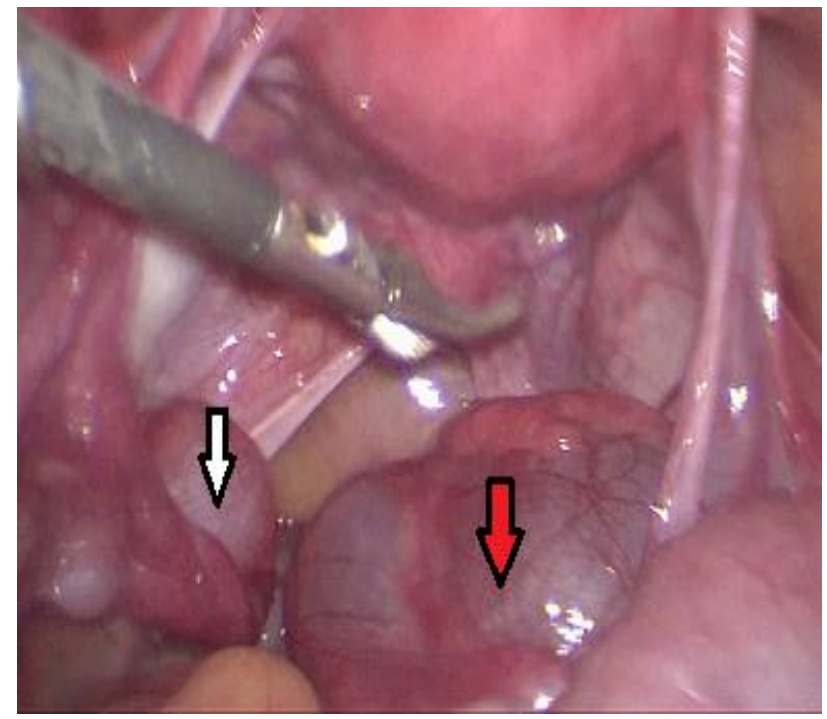

Fig 2: laparoscopy image with uterus and bilateral paratubal cyst on both right (red arrow) and left side (white arrow).

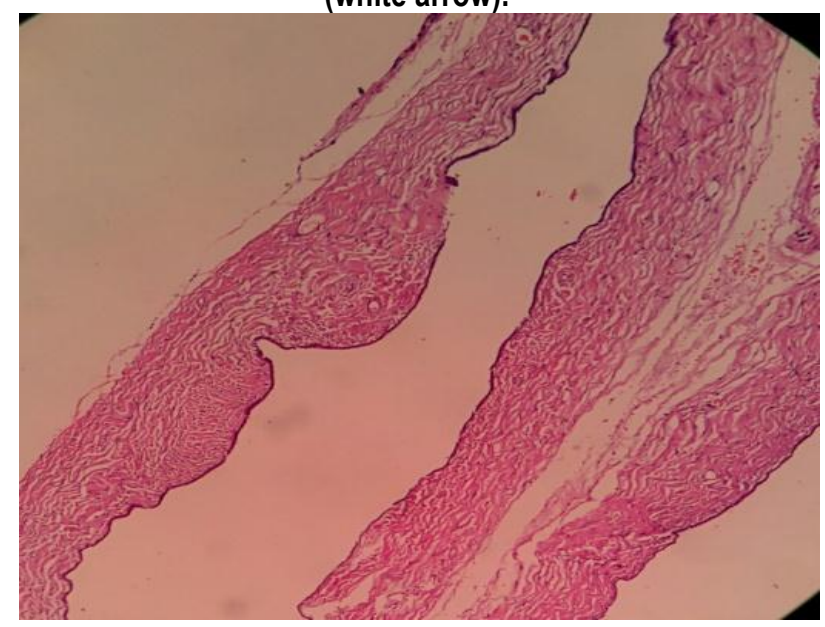

Fig 3: Histopathological showing benign paratubal cyst
The pathogenesis ${ }^{6}$ of IFTT has been explained as mechanical obstruction of adnexal veins and lymphatics, leading to pelvic congestion and edema with subsequent enlargement of the fimbrial end, thus leading to partial to complete torsion of the involved tube. Since vascular supply to the fallopian tubes comes from both ovarian and uterine vessels, so it leads to isolated tubal torsion without vascular compromise of the ovary.

IFTT is usually unilateral and more frequently diagnosed on the right side. The possible explanations for this are presence of sigmoid colon on left side, more common surgical exploration on right side with appendicitis as differential diagnosis and due to variance in venous return flow rate between the right and left fallopian tubes. In the case reported here also, torsion was found on right side.

The clinical presentation of IFTT is non-specific and hence a challenge for the clinician. Most of the cases present with acute abdomen with nausea and vomiting. Low grade pyrexia may also be seen. On clinical examination, abdominal tenderness is usually found with or without peritoneal signs. Laboratory findings are usually normal but in few cases, leukocytosis, raised sedimentation rate and $\mathrm{C}$-reactive protein can be found.

Ultrasound is often the first imaging modality done in a case of acute abdominal pain. The sonographic findings are of IFTT are dilated tube with thickened echogenic walls, normal-appearing uterus and ovaries with normal blood flow and free fluid in pouch of Douglas. These features are seen in most of the cases of IFTT but they are not pathognomic for the diagnosis?

Delayed presentation, nonspecific features and misdiagnosis of IFTT can lead to various complications such as fallopian tube necrosis and gangrenous transformation, leading to an increased risk for super infection and peritonitis. Local necrosis can also result in irreversible damage to the ipsilateral ovary ${ }^{8}$. To prevent this complication, prompt diagnosis of torsion and management is essential.

As the time duration between onset of pain and surgery increases, chances of saving the tubes decreases. In 2008, Harmon et al ${ }^{9}$ conducted a retrospective study of 8 IFTT cases and found the mean time taken from initial imaging to surgery was 1.67 days, because of which tube could not be salvaged in any of the cases. Laparoscopy is the now recommended as the gold standard in the management of fallopian tube torsion. It offers an added advantage of not only diagnosing the pathology but also simultaneous detorsion of adnexa to re-establish the blood supply so as to salvage the tube.

Cohen $S$ et al ${ }^{10}$ has done a study in 2003 to compare the role of laparoscopy to laparotomy for management of tubal torsion. They found that Laparoscopy is the most specific diagnostic tool for evaluating torsion and its simultaneous management. Laparoscopy is preferred over laparotomy since recovery after laparoscopy is faster, and it causes fewer pelvic adhesions, which is particularly important for women of reproductive age, who wish to preserve their fertility.

Treatment options of the isolated tubal torsion include either detorsion or salpingectomy, depending on the timing of surgical intervention and presence of complications. Tubal conservation should be favoured if possible but due to delay in diagnosis, it is often difficult to salvage the tubes. Here we have managed the case conservatively by laparoscopic detorsion and removal of paratubal cyst. 


\section{CONCLUSION}

Diagnosis of isolated fallopian tube torsion is a difficult task which requires high degree of clinical suspicion. Radiological investigation is helpful in its diagnosis but Laparoscopy is the gold standard for its evaluation and management. Conservative management including detorsion with cystectomy is the preferred treatment. Early diagnosis and timely management should be done to preserve the reproductive function.

\section{REFERENCES}

1. Gaied F, Emil S, Lo A, Baird R, Laberge JM. Laparoscopic treatment of isolated salpingeal torsion in children: case series and a 20-year review of the literature. J Laparoendosc Adv Surg Tech A. 2012;22:941-947.

2. Ahn JH, Chung J, Lee TS. Successful laparoscopic surgery for14-month-old infant with ovarian torsion. Obstet Gynecol Sci 2014; 57(2): 160-163.

3. Bland-Sutton T. Salpingitis and some of its effects. Lancet. 1890; 2: 1146.

4. Casey RK, Damle LF, Gomez-Lobo V. Isolated fallopian tube torsion in pediatric and adolescent females: a retrospective review of 15 cases at a single institution. J Pediatr Adolesc Gynecol. 2013 Jun;26(3):189-92.

5. Djing DC, Hsu S, Kao SP. Isolated torsion of hydrosaplinx in a postmenopusal woman. JSLS 2007 Apr-Jun;11(2):252-4.

6. M. P. Bondioni, K. McHugh, and L. Grazioli, "Isolated fallopian tube torsion in an adolescent: CT features," Pediatric Radiology, 2002; 32: 8: 612-613.
7. Volkan Sarper Erikci, Münevver Hosgor. Isolated salpingeal torsion in children: a case series and review of the literature. Ulus Travma Acil Cerr Derg January 2014; 20:1:75-8

8. Bernardus RE, Van der Slikke JW, Roex AJ, Dijkhuizen GH, Stolk JG. Torsion of the fallopian tube: some considerations on its etiology. Obstet Gynecol 1984; 64:675-678

9. Harmon JC, Binkovitz LA, Binkovitz LE. Isolated fallopian tube torsion: sonographic and CT features. Pediatr Radiol 2008;38:175-9.

10. Cohen S, Wattiez A, Seidman D, Goldenberg M, Admon D, Mashiach $S$ et al. Laparoscopy versus laparotomy for detorsion and sparing of twisted ischemic adnexa. JSLS. 2003; 7:295-9.

\section{Source of Support: Nil.}

Conflict of Interest: None Declared.

Copyright: (c) the author(s) and publisher. IJMRP is an official publication of Ibn Sina Academy of Medieval Medicine \& Sciences, registered in 2001 under Indian Trusts Act, 1882.

This is an open access article distributed under the terms of the Creative Commons Attribution Non-commercial License, which permits unrestricted non-commercial use, distribution, and reproduction in any medium, provided the original work is properly cited.

Cite this article as: Nidhi Jain, Rahul Manchanda, Sravani Chithra, Anshika lekhi. Isolated Fallopian Tube Torsion: A Rare Occurrence. Int J Med Res Prof. 2016; 2(3):210-12. 